\title{
The temporal fluctuation of the inverse participation ratio for localized field modes in three-dimensional percolation system
}

\author{
E. Martínez-Sánchez ${ }^{a}$, A. Díaz-de-Anda ${ }^{b}$, G. Burlak ${ }^{c}$, and R. Muñiz-Valdez ${ }^{a}$ \\ ${ }^{a}$ Facultad de Ingeniería, Universidad Autónoma de Coahuila, \\ Blvd. Fundadores Km. 13 Ciudad Universitaria, Arteaga, Coahuila 25354, Mexico, \\ ${ }^{b}$ Instituto de Física, Benemérita Universidad Autónoma de Puebla, \\ P.O. Box J-48, 72570 Puebla, Puebla, Mexico, \\ ${ }^{c}$ Centro de Investigación en Ingeniería y Ciencias Aplicadas, Universidad Autónoma del Estado de Morelos, \\ Av. Universidad 1001, Col. Chamilpa, 62210, Cuernavaca, Morelos, México.
}

Received 26 August 2020; accepted 19 November 2020

\begin{abstract}
We investigate the structure of the optical field radiated by the disordered optical nano-emitters randomly incorporated in three-dimensional cluster of a percolation material. Our numerical studies shown that the temporal variations of the inverse participation ratio allow analyzing the extended and localized field structures over a long time range. The properties of IPR and the dynamics of the lasing emitters allow to find the characteristic time scales when the localization of the field in a general three-dimensional disordered system occurs. The studied effect opens new perspectives to control the optical fields localization in modern optical nano-technologies.
\end{abstract}

Keywords: Optics localization; spanning cluster; random pores; incorporated nanoemitters; FDTD.

PACS: 63.20.Pw; 07.05.Tp; 61.43.Bn; 42.68.Ay; 42.25.Bs; 42.55.Zz

DOI: https://doi.org/10.31349/RevMexFis.67.285

\section{Introduction}

The localization of optical fields in disordered materials is one of the most interesting new concepts of optics. This type of localization is related to the absence of diffusion in random materials as a result of interference of all the scattered waves [1]. In disordered optical materials, the multiple scattering of light and the interferences between propagating waves lead to the formation of electromagnetic modes, in particular in three dimensions can be localized or extended depending on the degree of disorder and other factors. Recent studies have shown the possibility of creating useful optical structures by intrinsic disorder in diverse materials $[2,3]$. These structures are planned to be more economical and require relatively simple technology. This has increased the need to create favorable systems for light localization [4-10].

Disordered structures have been studied for investigation of complex optical phenomena, including light localization and random lasing [11-13].

Random lasers are mirror-less lasing systems that use highly disordered materials to obtain laser action [13]. Several models of random laser are available, e.g., diffusive feedback model, that has been applied to obtain absorption curves and energy velocities [14]. Transfer matrix model [15] has applications on the calculations of laser frequencies, threshold and spatial distribution of laser modes. Hakan et al. applied time-independent Maxwell-Bloch equations coupling the electrical field equations, polarization and inversion on a gain media. Similar approaches are generally used to study low dimensional systems [16-18].
Since the classical diffusive model cannot correctly describe the photon propagation on a dispersive gain media or with non-uniformly distributed loss [19], in this work we propose the use of the vectorial Maxwell-Bloch equations based on the three-dimensional (3D) Finite-difference time-domain (FDTD) method [20,21], instead of a diffusive approach.

Many works on 3D systems are focused on finding the critical disorder $[9,10]$, fractal dimension of critical modes $[22,23]$, as well as extended states [24] and localized. This last has been discussed on the photon free path regime for the strong [25-27] and weak [28] localization cases.

Previous efforts have concentrated on finding the complete characterization that encompass longitudinal and time scales [29]. We found very few studies about the time dynamics of random lasers and their correlations with inverse participation ratio (IPR) on the optical localization regime. For example, in [9], the relation of IPR was calculated to evaluate quantitatively the localization degree of a field in a 3D system. In that work the existence of light localization near the percolation threshold was proven. However, the study was performed for a time that does not exceed the critical time of lasing percolation. Our work could be an extension of that result in the scope of weak localization, in which we show that is possible to obtain localization on a wide temporal range, under and above of the percolation threshold of a 3D system.

In this paper we study the optical radiation of nanoemitters incorporated into 3D structures, where the spanning cluster serves as a set of bonds linking the pores of random size through which the field radiation of nanosources can flow .

The study of IPR behavior allows to relate the number of sites that participate in the field eigenstates [30]. Our treatment of the time dynamics of the field, through the 
Maxwell-Bloch equations coupled to the levels of emitters, besides IPR, permit to situate spatial and temporally the optical modes on the 3D system. This is one of most important integral characteristics of the optical field structure.

This paper is organized as follows. In Sec. 2 we formulate our basic model, approaches and the equations to study the structure of radiation field associated with nano-emitters incorporated in 3D percolating cluster. In Sec. 3 we present our numerical finite-difference time-domain method results for the properties of the field localization radiated by the emitters. Finally, in Sec. 4 we present our conclusions.

\section{Basic considerations and equations}

We consider a three-dimensional disordered cluster system, where the clusters are filled by an active media composed by light emitters. In such a non-uniform spatially disordered structure the radiating and scattering of field occurs in an incoherent way. The advantage of the time-dependent model is that one has access in principle to the full nonlinear dynamics of the laser system.

We follow the approach as in Ref. [31] to calculate the wave propagation in random media with gain. This model combines semiclassical laser theory with Maxwell's equations as follows. Incorporating a well-established FDTD, this model describes the coupling of emitter population rate equations (with different levels) to the field equations. Also, we calculate the integral emission of electromagnetic energy flux $I$ from a cubical sample $(x, y, z)$, where $x, y, z \in\left[0, l_{o}\right]$, and $l_{o}$ is a typical size for the nano system structures $l_{o}=10^{-4}$ $\mathrm{m}$.

We solve numerically the following equation

$$
\frac{\partial^{2} \mathbf{P}}{\partial t^{2}}+\Delta \omega_{a} \frac{\partial \mathbf{P}}{\partial t}+\omega_{a}^{2} \mathbf{P}=\frac{6 \pi \epsilon_{0} c^{3}}{\tau_{21} \omega_{a}^{2}}\left(N_{1}-N_{2}\right) \mathbf{E} .
$$

Here, we couple the polarization density $\mathbf{P}$, the electric field $\mathbf{E}$, and occupations of the levels of emitters, to find the optical emission from the system. In this equation, $\omega_{a}$ is the frequency of radiation, $\epsilon_{0}$ is the vacuum permittivity, $c$ is the velocity of light in vacuum, and $\tau_{21}$ is the decaying time from the second atomic level to the first one.

We find the electric and magnetic fields $\mathbf{E}$ and $\mathbf{H}$ from the Maxwell equations, together with the equations for the densities $N_{i}(\mathbf{r}, t)$ of atoms residing in $i$-th level [32]. In the case of lasers up to the fourth level, $i=0,1,2,3$, these rate equations read as (see [31] and references therein).

$$
\begin{aligned}
& \frac{\partial N_{3}(x, t)}{\partial t}=A_{r} N_{0}(x, t)-\frac{N_{3}(x, t)}{\tau_{32}}, \\
& \frac{\partial N_{2}(x, t)}{\partial t}=\frac{N_{3}(x, t)}{\tau_{32}}+\frac{\mathbf{j}(x, t) \cdot \mathbf{E}(x, t)}{\hbar \omega_{a}}-\frac{N_{2}}{\tau_{21}}, \\
& \frac{\partial N_{1}(x, t)}{\partial t}=\frac{N_{2}(x, t)}{\tau_{21}}-\frac{\mathbf{j}(x, t) \cdot \mathbf{E}(x, t)}{\hbar \omega_{a}}-\frac{N_{1}}{\tau_{10}}, \\
& \frac{\partial N_{0}(x, t)}{\partial t}=\frac{N_{1}(x, t)}{\tau_{10}}-A_{r} N_{0} .
\end{aligned}
$$

The lifetimes and energies of upper and lower lasing levels are $\tau_{21}, E_{2}$ and $\tau_{10}, E_{1}$, respectively. Therefore, the individual frequency of radiation of each emitter is $\omega_{a}=$ $\left(E_{2}-E_{1}\right) / \hbar$. An external source excites emitters from the ground level $(i=0)$ to third level $(i=3)$ at a certain rate $A_{r}$, which is proportional to the pumping intensity in experiments. After a short lifetime $\tau_{32}$, the emitters transfer nonradiatively to the second level. Emitters can decay from the upper (second level) to the lower level (first level) by both spontaneous and stimulated emission, here $(\mathbf{j} \cdot \mathbf{E}) / \hbar \omega_{a}$ is the stimulated radiation rate. Finally, emitters can decay nonradiatively from the first level back to the ground level. Below we consider the situation when the incipient percolating cluster is completely filled with light sources.

In calculations, we consider the gain medium with parameters close to GaN powder, similar to Ref. [33]. The lasing frequency $\omega_{a}$ is $2 \pi \times 3 \times 10^{13} \mathrm{~Hz}$, the lifetimes are $\tau_{32}=0.3 \mathrm{ps}, \tau_{10}=1.6 \mathrm{ps}, \tau_{21}=16.6 \mathrm{ps}$, and the dephasing time is $T_{2}=0.0218$ ps. Suppose that in each node of the network there are many emitters, in such a way that the total density of emitters inside the percolation cluster being $N=N_{0}+N_{1}+N_{2}+N_{3}=3.3 \times 10^{24} \mathrm{~m}^{-3}$. The refractive index of a host material is $n=2.2$ that is close to the typical values for ceramics $\mathrm{Lu}_{3} \mathrm{Al}_{5} \mathrm{O}_{12}, \mathrm{SrTiO} 3, \mathrm{ZrO}_{2}$, see review [34]. To simulate the noise in our system the initial seed for the electromagnetic field has been created with random phases at each node. In the structure all internal uncoupled clusters have been omitted. The percolation cluster has a sponge structure, that depends on the actual random sampling, re-running simulation with another random seed value will lead to percolation cluster with somewhat different geometry.

The inverse participation ratio is the average of the fourth power of the wave function and it is a convenient quantity which distinguishes the extended state from the localized state [35]. To evaluate quantitatively the degree of field localization, we calculate the inverse participation ratio defined as

$$
I=\frac{l_{o}^{3} \int|\mathbf{E}|^{4} d^{3} r}{\left(\int|\mathbf{E}|^{2} d^{3} r\right)^{2}},
$$

For small values of the IPR the field extends over the entire cubic network. Nevertheless, there is a minimum critical value of the IPR above from there the localization of the field in the medium exists, as confirmed in Ref. [9] with the FDTD numerical simulations. Also in Ref. [9] it is observed that $I(p) \sim 0.5$ up to percolation transition zone for $p<p_{c}$ and $I(p)$ changes sharply at $p \simeq p_{c}$ where the percolating phase transition occurs. Here, we focus our attention on the calculation of the IPR for fixed value of $p=0.49$. Our motivation to use $p=0.49$ is simple, for this value of $p$, we already pass the critical percolation value $p_{c}=0.318$, and we have almost the same probability of having pores or material. 


\section{Numerical results}

There are many ways to add disorder to a sample. In our study, in addition to the parameter $p$ defined as the probability of pore occupation, we have added randomness to the radio of the pores, similar to the approach made in Ref. [36]. We characterize the radius $r$ as a random variable function uniformly distributed in the interval $[a, b]$ where $a=[1-\alpha]$ and $b=[(1+\alpha) R]$, for $0<\alpha<1$ and $R>0$ is the average radius, $\sigma^{2}=R^{2} \alpha^{2} / 3$ is the standard deviation for $r_{i}$, and $r_{i}$ is the radius of $i$ pore, for more details see [36].

In what follows we simulate the radiated field distribution by FDTD techniques for indicated parameters. The sequence of the simulations is as follows: i) calculating the geometry of the percolation cluster for $p=0.49$ and average radius $R=0.49$; ii) calculating the photon field $\mathbf{E}$ generated by spatial nanosources incorporated in this cluster deals with the use FDTD techniques [21]; iii) obtain the dynamics of the emitters Eqs. (2.5) and the energy flow $I$, such that $I=\oint_{s}(\mathbf{K} \cdot n) d s$, where $\mathbf{K}$ is the Pointing vector and $\mathbf{n}$ is the normal unit vector to the surface $s$ of the cube; finally iv) calculation of IPR Eq. (6), for each simulation time. In our calculation the following dimensionless variables are used: $r \rightarrow / l_{0}, t \rightarrow\left(c / l_{0}\right)$, where $l_{0}$ is the size of the system and $c$ is the velocity of light in vacuum. Our results are shown in Figs. 1-6.

Figure 1 shows the dynamics of levels $N_{1}$ and $N_{2}$, as well as the energy flow $I_{x}$ in the $x$ direction, when $\sigma=0.04$. After an initial excitation time a large number of emitters are synchronized to contribute to the stimulated radiation, this is observed in the $I_{x}$ curve (blue line), which shows some oscillations over time, these oscillations are the result of the
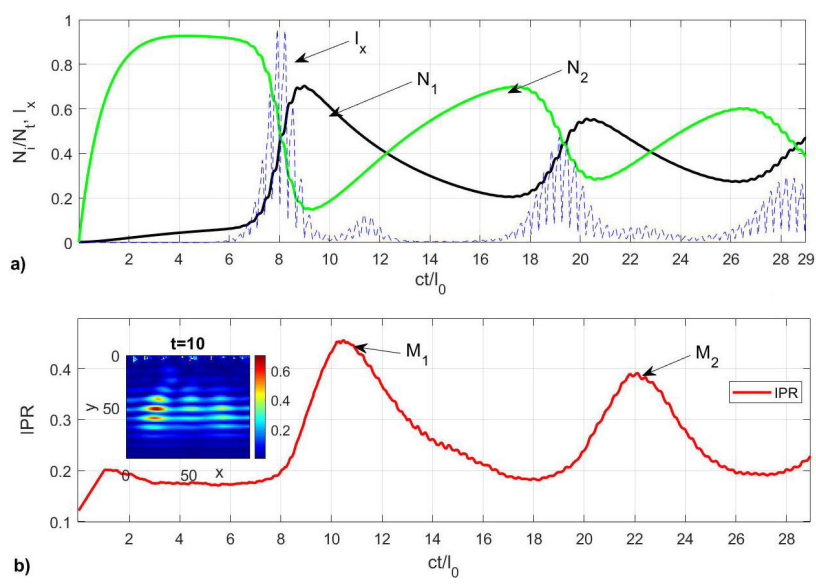

FIGURE 1. Time dynamics of the field and lasing level populations obtained from the numerical FDTD simulations of the system for parameters $L=100$ and $\sigma_{r}=0.04$. a) The dotted blue line shows the energy flow $I_{x}$ in the $x$ direction as the function of time, the lasing levels $N 1$, and $N_{2}$ populations are displayed in black and green line respectively. b) The average IPR as function of time $\left(10^{-9} \mathrm{~s}\right)$. The inset exhibits the amplitude of the localized field mode in output $x=25, y=50$ for time 10 .

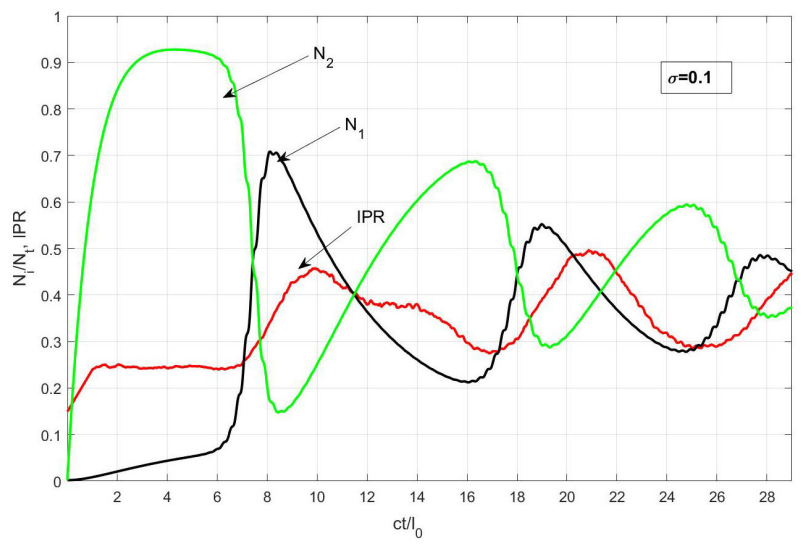

FIGURE 2. (Color online) Dynamics of the population of lasing levels $N_{1,2}$ (green and black lines respectively) and the IPR (red line) as functions of time for $\sigma=0.1$. We observe that the IPR has periodical fluctuations (maximal values) related to the field localization in times $t=10,21$ and 29 .

inhomogeneity of the structure. We can clearly see that $I_{x}$ has three maxima over time, specifically at times $t=8,19$ and 28.

When an excitation is applied, this causes the atoms initially in the lower energy level $\left(N_{1}\right)$ to begin stimulated transitions or "jumps" up to the higher energy level, so we can observe that the first oscillation of the $N_{1}$ level ( black curve) is ascending near time $t=6$. At the same time, the same applied excitation will also cause any atom initially at the higher energy level $\left(N_{2}\right)$ to start making stimulated transitions and their energy jump down at a rate that is proportional to the intensity of the applied signal, multiplied by the number of atoms at the initial level (that is, higher). We can observe the above in the initial behavior of $N_{2}$.

At the intersection of both levels $\left(N_{1}\right.$ and $\left.N_{2}\right)$ the maximum amount of energy emerges in the percolation cluster, this is why at $t=8$ we observe the greatest amplitude for $I_{x}$. The same goes for times $t=18$ and 28, but with smaller amplitudes.

Figure 1b) shows the dynamics of the IPR as a function of time, we can see two maxima in the IPR, labeled as $M_{1}$ at $t=10.5$ and $M_{2}$ at $t=22$. In a neighborhood of $M_{1}$ we found a dominant mode with localized field amplitude for a time $t=10$. However, in the vicinity of $M_{2}$ there has not been enough accumulated energy to obtain a localized field, this is because at that point the IPR $\sim 0.38$, which is not sufficient to achieve the effects of localization in the environment, as explained in Sec. 2.

Figure 2 shows the dynamics of $N_{1}, N_{2}$ and the IPR as a function of time for $\sigma=0.1$. We can observe that the IPR reaches its maximum values with the possibility of finding a field localized at times $t=10,21$ and 29; these maxima of the IPR are associated with the decay of the $N_{2}$ level. By conservation of energy, the radiation must flow somewhere, and this is reflected in the elevation of the IPR values. Above the graphic of the IPR (in red), we can observe the modes localized in the vicinity of the aforementioned times; these, modes are shown in Fig. 3. 
a) $t_{9}, z=28$

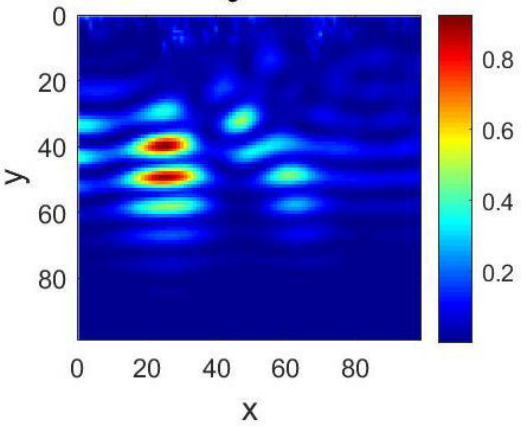

d) $t_{10}, z=25$

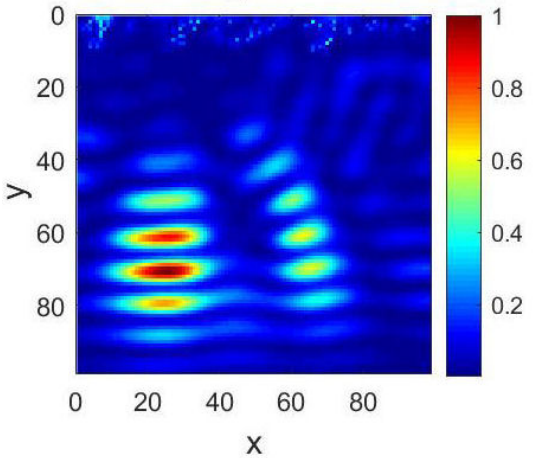

b) $t_{20}, z=28$

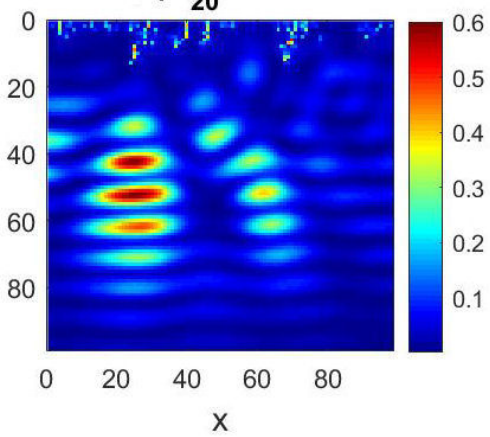

e) $t_{21}, z=22$

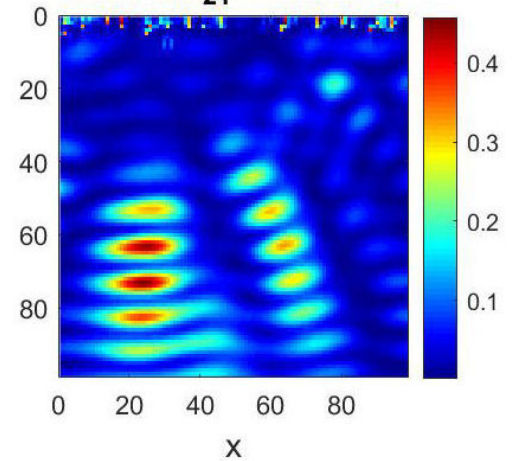

c) $t_{28}, z=40$

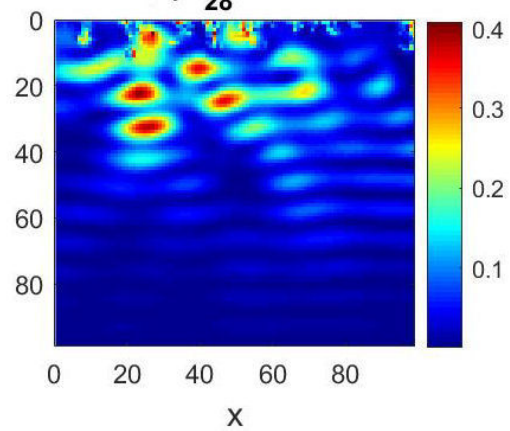

f) $t_{29}, z=26$

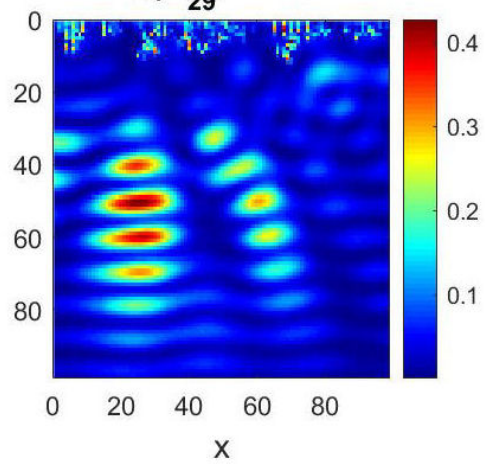

FIgURE 3. (Color online) The spatial distribution of field $\left|E_{x}\right|$ for the parameters shown in Fig. 2 at different times. From panels a)-f) we observe various spots corresponding to the modes localized with large amplitude in the area free of emitters.

Figure 3 shows the distribution of the $\left|E_{x}\right|$ field in some times for the results shown in Fig. 2. In Fig. 3, we can see some circles with a field width localized for different times. Here $z$ represents one of the 3D system planes for a better viewing. We want to note that there is no single time for which we can find a localized field, but in the vicinity of the maximum (IPR) $\sim 0.5$, the probability of finding high amplitude modes is very close to one, as we show below.

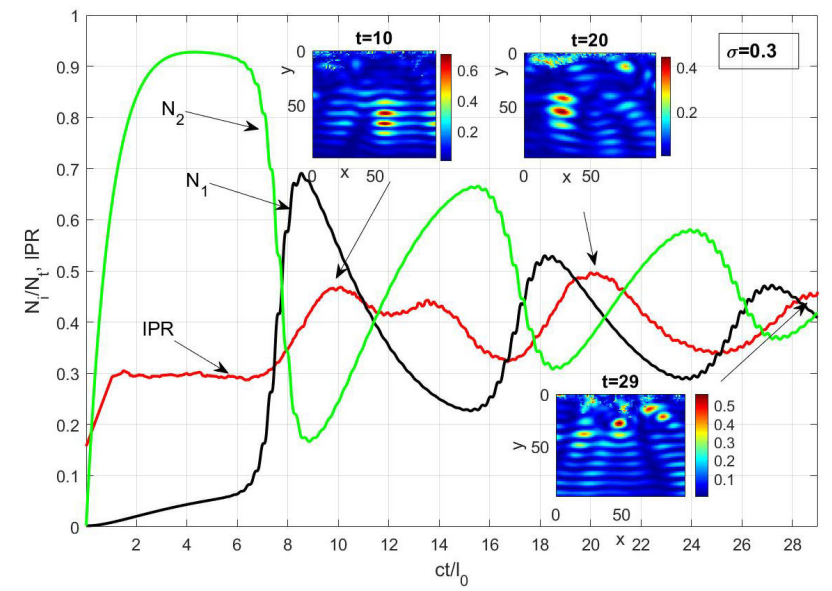

FIGURE 4. (Color online) Dynamics of laser levels populations $N_{1}, N_{2}$ and IPR for $\sigma=0.3$. The insets show that the peaks of IPR shown in Fig. 3b) correspond to the 3D localized field modes in the times where the IPR $\sim 0.5$, these times are $t=10,20$ and 29 .
In Fig. 4, the same as in Fig. 2 is shown, but now $\sigma=0.3$. In this situation, percolation has not yet occurred as in the previous cases. The internal panels show some planes of the $3 \mathrm{D}$ system with modes localized in the times where the IPR $\sim 0.5$, these times are $t=10,20$ and 29 .
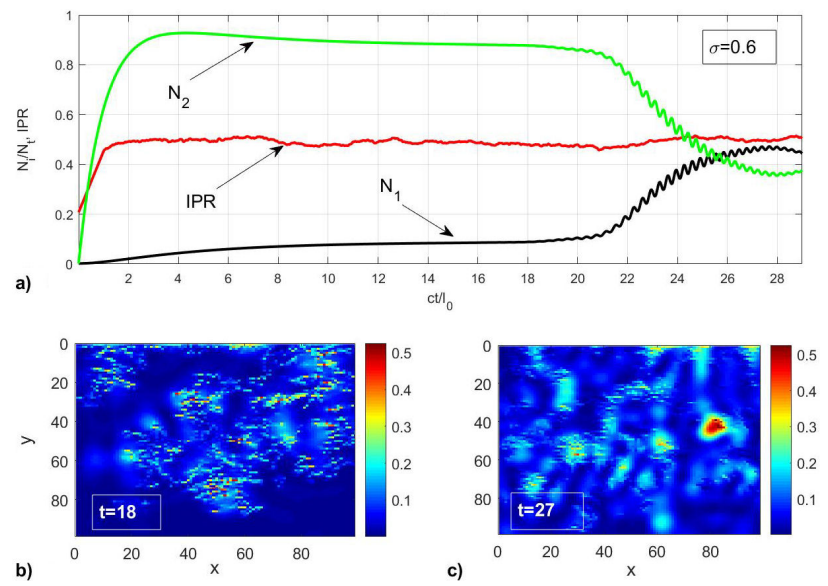

FIGURE 5. (Color online) Numerical simulations made for system cubic network of size $L=100$ for $\sigma_{r}=0.6$. a) Average of the IPR calculation as a function of time, (red line). Densities of the lasing levels $N_{1}$, and $N_{2}$, black and green line respectively. b) Distribution of the $\left|E_{x}\right|$ field at time $t=18$, the spot color boxes show the field in the position of the emitters. c) Distribution of the $\left|E_{x}\right|$ field at time $t=27$, the point around $x=85, y=40$ exhibits the localized field amplitude. 


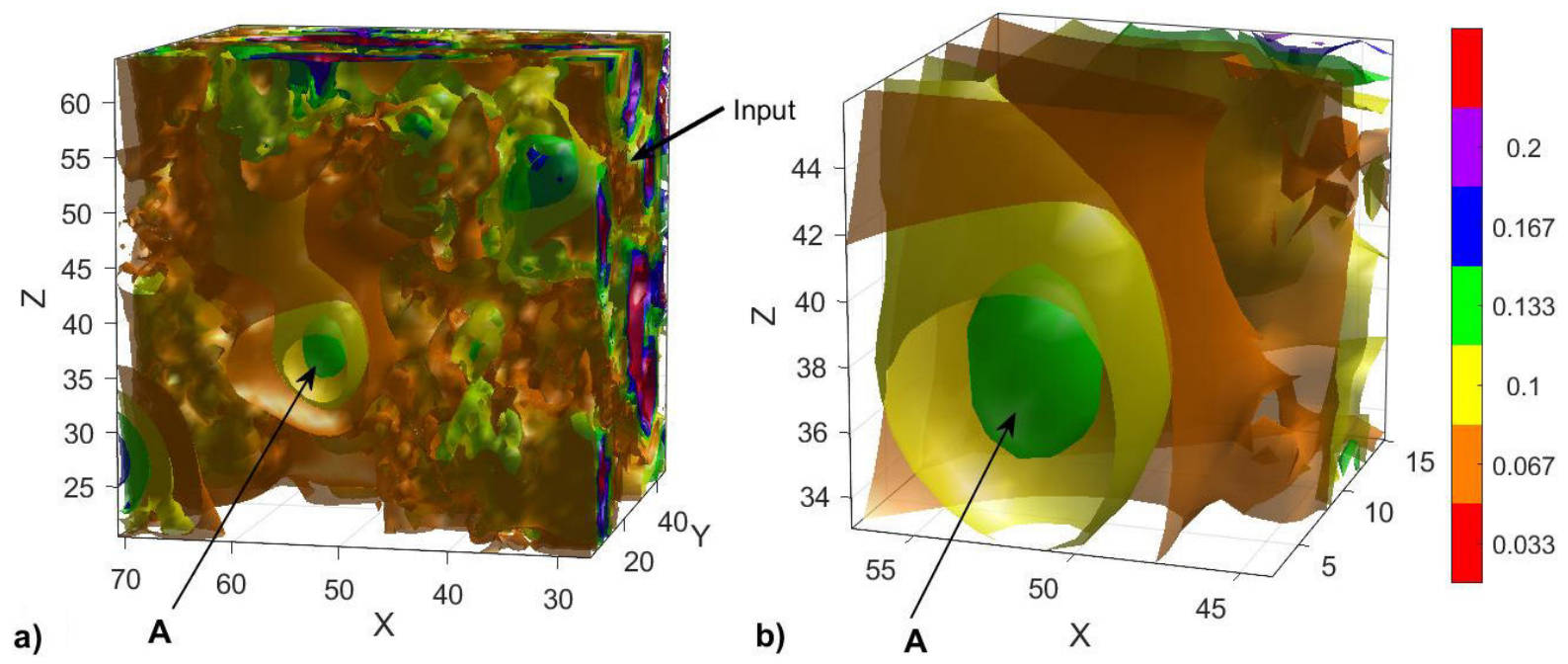

Figure 6. (Color online) Amplitude of the field $\left|E_{x}\right|$, generated by the emitters incorporated in the percolating cluster at $\sigma=0.6$. Arrow $A$ shows one of the localized field mode in the 3D cube. b) Same as in a), but with a more detailed view, where one of the localized modes indicated by the symbol $A$ is displayed in part of $3 \mathrm{D}$ cube.

We want to compare what happens in the sample with incorporated nanoemitters once the percolation has occurred. This situation is shown in Fig. 5, where we have used $\sigma=0.6$, a value for which a large cluster is formed that spans the entire network. In Fig. 5a, we can see that there is a longer synchronization time of the laser levels; this is because the number of pores in the system has increased considerably and therefore the volume has increased. Near time $t=28, N_{2}$ reaches its minimum value. It is interesting that the IPR in this situation is a constant value $\sim 0.5$, so we would expect that for each time there would be localization of the field. However, in Fig. $5 \mathrm{~b}$ for a time $t=18$, we can observe the positions of the emitters in the sample, and the cooresponding amplitude of the field $E_{x}$ without presenting a localized field. On the other hand, for a time $t=27$, after the laser generation has occurred, we observe some mode in the position $x=80, y=40$ (Fig. 5c) with localized field amplitude, other modes appear in the sample but with less amplitude. We can conclude that in order to obtain a field localized at different times, it is necessary and sufficient that the IPR reaches maximum values of approximately 0.5 , and the laser generation occurs in the percolation cluster.

Figure 6 shows the isosurface of the amplitude of the $E_{x}$ $3 \mathrm{D}$ field. In this case the field is generated by the emitters incorporated in the percolation system with $\sigma=0.6$. Figure 6 a clearly shows some inclusions in blue color occupying different parts of the sample. Figure $6 \mathrm{~b}$ shows a detailed view of Fig. 6a. We can see that the localized field structure is localized beyond the source area (indicated by the symbol A), whose shape is normally ellipsoidal, coinciding with the characteristic property of finding closed cycles in the optical localization.

One drawback of our proposed method is based on the huge quantity of computational resources needed to work
3D problems. To overcome these limitations, different approaches such as solving the wave equation in the frequency domain could be used, see [37].

\section{Conclusions}

We have investigated the structure of the optical field radiated by the disordered nano-emitters randomly incorporated in three-dimensional cluster of a percolation material. Our numerical studies shown that the temporal variations of the inverse participation ratio allow analyzing the extended and localized field structures over a long time range. The properties of IPR and the dynamics of the lasing emitters allow to find the characteristic time scales when the localization of the field in a general three-dimensional disordered system occurs.

Studied conditions are considered for a general case and thus, are independent of the occurrence/absence of the percolation in system. Due to this study, one can evaluate the times in which the localized modes of maximum amplitude are recovered in the 3D system. The combination of localization and random lasing has practical consequences because every individual random-laser source would give a unique emission spectrum defined by the specific localized modes in each sample. Such modes can be used as resonators to add functionality to photonic components. The studied effect opens new perspectives to control the optical fields localization in modern optical nano-technologies.

\section{Acknowledgements}

This work was supported in part by VIEP-BUAP under Project ID 00255-VIEP2019, and by CONACYT (México) under the grant No. A1-S-9201. 
1. D.S. Wiersma et al., Localization of light in a disordered medium, Nature 390 (1997) 671, https : / / doi.org/10. $1038 / 37757$

2. D. Vollhardt, Localization Effects in Disordered Systems. Festkiirperprobleme 27 (1987) 63.

3. D.S. Wiersma, Disordered photonics, Nat. Photonics 7 (2013) 188, https://doi.org/10.1038/nphoton. 2013.29

4. D. Vollhardt, P. Wolfle, Scaling Equations from a SelfConsistent Theory of Anderson Localization, Phys. Rev. Lett. 48 (1982) 699, https://doi.org/10.1103/ PhysRevLett. 48.699

5. P. Sebbah, D. Sornette, C. Vanneste, Anomalous diffusion in two-dimensional Anderson-localization dynamics, Phys. Rev. B 48 (1993) 12506. https://doi.org/10.1103/ PhysRevLett.48.699

6. M.M. Sigalas et al., Localization of electromagnetic waves in two-dimensional disordered systems, Phys. Rev. B. 53 (1996) 8340. https://doi.org/10.1103/PhysRevB. 53.8340

7. T. Schwartz et al., Transport and Anderson localization in disordered two-dimensional photonic lattices, Nature 446 (2007) 52, https://doi.org/10.1038/nature05623

8. F. Riboli et al., Anderson localization of near-visible light in two dimensions, Opt. Lett. 36 (2011) 127 https://doi. org/10.1364/OL.36.000127

9. G. Burlak, E. Martinez-Sanchez, The optical Anderson localization in three-dimensional percolation system, Opt. Commun. 387 (2017) 426, https://doi.org/10.1016/j. optcom.2016.10.068

10. C. Conti, A. Fratalocchi, Dynamic light diffusion, threedimensional Anderson localization and lasing in inverted opals, Nature Phys. 4 (2008) 794, https://doi.org/10. $1038 /$ nphys 1035

11. P. Sebbah, C. Vanneste, Random laser in the localized regime, Phys. Rev. B, 66 (2002) 144202, https://doi.org/10. 1103 /PhysRevB.66.144202

12. H. Noh et al., Control of lasing in biomimetic structures with short-range order, Phys. Rev. Lett. 106 (2011) 183901, https : //doi.org/10.1103/PhysRevLett.106.183901

13. D. S. Wiersma, The physics and applications of random lasers, Nat. Phys. 4 (2008) 359, https://doi.org/10.1038/ nphys 971

14. M. Gaio, M. Peruzzo and R. Sapienza, Tuning Random Lasing in Photonic Glasses, Opt. Lett. 40 (2015) 1611, https : //doi.org/10.1364/OL.40.001611

15. N. Bachelard, J. Andreasen, S. Gigan and P. Sebbah, Taming Random Lasers Through Active Spatial Control of the Pump, Phys. Rev. Lett. 109 (2012) 033903, https://link.aps.org/doi/10.1103/ PhysRevLett.109andreasen2011

16. J. Andreasen et al., Modes of random lasers, Adv. Opt. Photon. 3, (2011) 88, https: //doi.org/10.1364/AOP.3. 000088
17. C. Vanneste, P. Sebbah, and H. Cao, Lasing with Resonant Feedback in Weakly Scattering Random Systems, Phys. Rev. Lett. 98 (2007) 143902, https://link.aps.org/doi/ $10.1103 /$ PhysRevLett.98.143902

18. P. Sebbah, and C. Vanneste, Random laser in the localized regime, Phys. Rev. B, 66 (2002) 144202, https://link. aps.org/doi/10.1103/PhysRevB.66.144202

19. M. A. Noginov, J. Novak, D. Grigsby, and L. Deych, Applicability of the diffusion model to random lasers with non-resonant feedback, J. Optics A: Pure and Applied Optics, 8, (2006) S285, https://iopscience.iop.org/ article/10.1088/1464-4258/8/4/S31/meta

20. G. Burlak, Y.G. Rubo, Mirrorless lasing fromlight emitters in percolating clusters, Phys. Rev. A 92 (2015) 013812, https: //doi.org/10.1103/PhysRevA.92.013812

21. A. Taflove, S.C. Hagness, Computational Electrodynamics: The Finite-Difference Time-Domain Method, 3rd ed. (Artech House Publishers, Boston, London, 2005),pp. 51-327.

22. J. Bauer, T.-M. Chang, and J. L Skinner, Correlation length and inverse-participation-ratio exponents and multifractal structure for Anderson localization, Phys. Rev. B, 42 (1990) 8121, https://link.aps.org/doi/10.1103/ PhysRevB.42.8121

23. G. Burlak, The Dynamic Three-Dimensional Localization of Fields in Active Percolating Systems, Adv. in Math. Phys. 2019 (2019) 1, https://doi.org/10.1155/2019/ 5867012

24. S. Mujumdar, M. Ricci, R. Torre, and S. Wiersma, Amplified Extended Modes in Random Lasers, Phys. Rev. Lett. 93 (2004) 053903, https://link.aps.org/doi/10. 1103/PhysRevLett.93.053903

25. F. Scheffold, R. Lenke, R. Tweer and G. Maret, Localization or classical diffusion of light, Nature 398 (1999) 206, https://www.nature.com/articles/18347

26. A. A. Chabanov, M. Stoytchev, and A. Z. Genack, Statistical signatures of photon localization, Nature 404 (2000) 850, https://www.nature.com/articles/35009055

27. D. S. Wiersma, M. P. van Albada, B. A. van Tiggelen, A. Lagendijk, Experimental evidence for recurrent multiple scattering events of light in disordered media, Phys. Rev. Lett. 74 (1995) 4193, https://journals.aps.org/prl/ abstract/10.1103/PhysRevLett.74.4193

28. J. Fallert et al., Co-existence of strongly and weakly localized random laser modes, Nat. Photonics, 3 (2009) 279, https: //www.nature.com/articles/nphoton.2009.67

29. R. Sapienza, Determining random lasing action, Nature Rev. Physics, 1 (2019) 690, https: / /www . nature.com/ articles/s42254-019-0113-8

30. F. Wenger, Inverse Participation Ratio in $2+\epsilon$ Dimensions, Zeitschrift fur Physik B Condens. Matter 36 (1980) 209, https://doi.org/10.1007/BF01325284

31. X. Jiang, C.M. Soukoulis, Phys. Rev. Lett. 85 (2000) 70, https://doi.org/10.1103/PhysRevLett.85.70 
32. A.E. Siegman, Lasers, (University Science Books, Mill Valley California, 1986), pp. 27-39.

33. H. Cao et al., Random Laser Action in Semiconductor Powder, Phys. Rev. Lett. 82 (1999) 2278, https://doi.org/10. 1103/PhysRevLett.82.2278

34. J. Sanghera et al., Ceramic Laser Materials, Materials 5 (2012) 258, https://doi.org/10.3390/ma5020258

35. H. Shinobu, Localization Length and Inverse Participation Ratio of Two Dimensional Electron in the Quantized Hall Effect,
Prog. Theor. Phys. 76 (1986) 1210, https://doi.org/ $10.1143 /$ PTP. 76.1210

36. G. Burlak, Y. Calderon-Segura, Percolation and lasing in real 3D crystals with inhomogeneous distributed random pores, Phys. B. 453 (2014) 8, https://doi.org/10.1016/j. physb.2014.04.030

37. H. Cao et al., Spatial confinement of laser light in active random media, Phys. Rev. Lett. 84 (2000) 5584, https://journals.aps.org/prl/abstract/ 10.1103/PhysRevLett.84.5584 